\title{
An Analysis of the Paradox of Constant Increasing Activity in Logistics Network Planning
}

\author{
Feng KONG, Bin LI \\ School of Economics and Management, \\ North China Electric Power University \\ Baoding, China \\ e-mail: 824334891@qq.com
}

\begin{abstract}
At present our country a lot of good logistics network, according to the user's own area can be related information through their own logistics network logistics network is to find the area logistics network based on the quantity, mainly to provide logistics information in the area. At present, most of the related theories are the paradox of constant increase and the paradox of the longest path length. In this paper, the method of minimum network constraint is used to prove that the maximum path length paradox does not exist in the logistics network planning.
\end{abstract}

Keywords-component; logistics network; maximum path length paradox; minimum network constraints

\section{INTRODUCTION}

With the development of Internet plus, people increasingly high demand for logistics, especially the logistics customer on the accuracy and timeliness requirements become more and more obvious, in order to meet customer requirements, we are planning for the practice of logistics network, one of the most advanced methods is the overlapping network, namely high abstraction and expression between different materials and they are linked to the most concise symbols, which uses nodes (or arrow) said some work with the arrow (or nodes) to express all kinds of work of a planned composition similar to the network in the form of the order and the relationship between the choice and expression of these symbols is because of its simplicity and image; then, through the logical relationship related accumulated find a set of work to determine the total time-consuming project, the logical order of this working group to form a "step chain" is called the key line; Then, by constantly balance the work consumption, shorten the critical path, in order to improve the network plan, the final choice a satisfactory solution into practice; when the program execution, using network planning control and continuous balance, so as to ensure the reasonable to make use of manpower and material resources and financial resources, obtain the biggest effect to pay a minimum.

However, there are still some paradoxes: the path length paradox, the paradox of constant increasing process

The paradox of long path: there is a paradox that there are "the same time constraints between different processes in the overlapping network, which may produce different maximum path length". This paper presents a new method to express the overlapping network by studying the cause of the paradox. This method is not only consistent with the classical CPM network, but also can be used to solve the time parameters and the critical path.

Paradox of constant increasing process: no matter whether a key process is shortened or extended, the total time limit is extended. This is the constant increase process in the overlapping network.

\section{A REVIEW OF RESEARCH STATUS}

After the Crandall put forward the overlapping network in 1973, it attracted the attention of scholars from various countries. Proposed by Terry in 2003, on the network can overlap single code standard conversion, different time constraints will be transformed into a variety of all time constraints, such as the S-S type time constraints, and gives the transformation method. In 1992, Elmaghraby put forward the AOA model of overlapping network, and used the method of mathematical programming to solve the time cost tradeoff problem in the network. Since 1998, the research content and structure of this paper has become the focus of research in the network with overlapping relationship. An optimization method of De Mohammad et al respectively, the branch and bound method is proposed to solve the scheduling problem in resource constraint in overlapping network under the network and solve the overlapping with cash flow and resource constrained project scheduling problem; and then again in 1999 when the proposed project scheduling problem in overlapping network with multi resource constraints. De, Kim K, and Fest discuss the exact algorithm for the problem. In this paper, a new branch and bound method is proposed to solve the scheduling problem with resource constraints in the presence of Ammar proposes a genetic algorithm to solve the scheduling problem of resource constrained scheduling problem in a network with cash flows. Terry $\mathrm{W}$ is constantly searching for the exact algorithm for the shortest total time limit of the resource scheduling problem under the constraint of resources in the general precedence network.

Study on overlapping network in China relatively late, China's export of overlapping network mainly focus on the overlapping network modeling method, in overlapping network time parameters and maneuvering time calculation method, to determine the key chain and how to use the double code network said these basic problems on overlapping network. Due to the wide application of the 
AOA network, some scholars are also committed to the establishment of AON linked network plan into a dual code network plan. Chinese scholars in this paper is a calculation method of AON overlapping network fuzzy duration.

However, in recent years, with the in-depth study of the time parameters of CPM network, the research on the "singular phenomenon" of the network time parameters. But the research literature in this field. Which is representative of the Wriest (1981), Kong Feng (2014), Qi Jianxun (2014) found in the feature of the length of the line of the key with the key process duration increased, but total duration shortened; the maneuver time becomes more "strange phenomenon or unusual characteristics". It is also found that there are similar phenomena in repetitive projects. In this paper, we also find out the key problem of the decomposition of the key process, that is to say, it is no longer the key process after the key process is decomposed into a sequence of FTS $=0$. These strange phenomena in the project management software (such as P3, MS Project, etc.) has also been the existence of proof.

The paradox of the constant increasing process in the logistics network planning

\section{A. An example}

The following example is given to illustrate the process of constant increase in the logistics network plan

Figure 1 shows that there are four key processes in the A-B-G-E-H network, which are A-B-E-H; A-B-E-F-H; A-B-G-E-F-H; the total time limit for the overlap network calculation is about 99 days

If the duration of the key process $\mathrm{E}$ in the overlapping network is increased by 1 day, that is, the duration is changed to a time of 12 days, the time parameter of the network diagram is recalculated as shown in figure 3-2:

Figure 2 shows that there is 1 key process in the network diagram for A-B-E-H; the total time limit for network computing is about 100 days. The total duration of the network increases with the increase of the duration of the key process.

If the duration of the $\mathrm{E}$ of the process is reduced by 1 day, the duration is changed to a period of 10 days, the time parameter of the network graph is recalculated as shown in figure 3-3

Figure 3 shows the network diagram of the key processes are two, respectively, A-B-E-F-H; A-B-G-E-F-H; total duration of 100 days.

Through the above network figure 1 Figure 2 Figure 3 shows that regardless of the key processes in the lap $\mathrm{E}$ shorten or extend the total duration are extended. This is the constant increase process in the overlapping network.

B. An Analysis of the Traditional Algorithm of the Constant Increasing Paradox in the Logistics Network Planning

In accordance with the traditional algorithm, as shown in
Figure 3-2 network, if the key process E duration increased by 1 days, to become a day of 12 ,

$$
\begin{aligned}
& E S_{E}=\max \left\{\begin{array}{c}
E F_{B}+F T S_{B E} \\
E F_{G}+F T F_{G E}-d_{E}
\end{array}\right\}=64 \\
& E F_{E}=\max \left\{\begin{array}{c}
E S_{B}+d_{B} \\
E F_{G}+F T F_{G E}
\end{array}\right\}=76
\end{aligned}
$$

While the initial network graph in 3-10 process $E$ the earliest finish time for seventy-fifth days, so the process of $\mathrm{E}$ increases the duration of 1 days, the total duration of postponed 1 days to 100 days.

As shown in Figure 3-2, if the critical process E duration is reduced by 1 day, it becomes a day of 10 days,

$$
\begin{aligned}
& E S_{E}=\max \left\{\begin{array}{c}
E F_{B}+F T S_{B E} \\
E F_{G}+F T F_{G E}-d_{E}
\end{array}\right\}=65 \\
& E S_{F}=\max \left\{\begin{array}{c}
E F_{C} \\
E S_{E}+S T S_{E}
\end{array}\right\}=73 \\
& E F_{E}=\max \left\{\begin{array}{c}
E S_{B}+d_{B} \\
E F_{G}+F T F_{G E}
\end{array}\right\}=75 \\
& E F_{F}=\left(E S_{F}+d_{F}\right)=88 \\
& E S_{H}=\max \left\{\begin{array}{c}
E F_{E}+F T S_{E H} \\
E F_{F}+F T F_{F H}-d_{H}
\end{array}\right\}=92,
\end{aligned}
$$

While the initial network process $\mathrm{H}$ the earliest start time for ninety-first days, therefore, the $\mathrm{E}$ process reduces the duration of 1 days, the total duration of postponed 1 days to 100 days.

Through the process of $\mathrm{E}$ analysis showed that the duration when the process of $\mathrm{E}$ gradually shortened, because the FTFGE constraints exist, the process of E's earliest start time postponed, resulting in the extension of time. But the process of the earliest start time is defined as a process at the earliest possible start time, that is to say the process $\mathrm{E}$ the earliest start time, and FTFGE, regardless of constraints, therefore, problems of logical relations and the calculation method of the overlapping network.

The problems mentioned above show that the traditional network computing theory and methods are flawed. It is our thinking of the traditional network is in trouble, is the lack of a systematic and accurate definition of the relevant concepts, leading to the failure to correctly identify key processes and key lines. It is also the correct application of the traditional network is limited.

\section{Modification OF THE PARAdOX OF CONSTANT INCREASING PROCEDURE}

By adjusting the duration of the process (Yan Chang), the paradox of the constant increasing procedure is modified, which proves that the paradox of the constant increasing process does not exist in the minimal constraint network. 


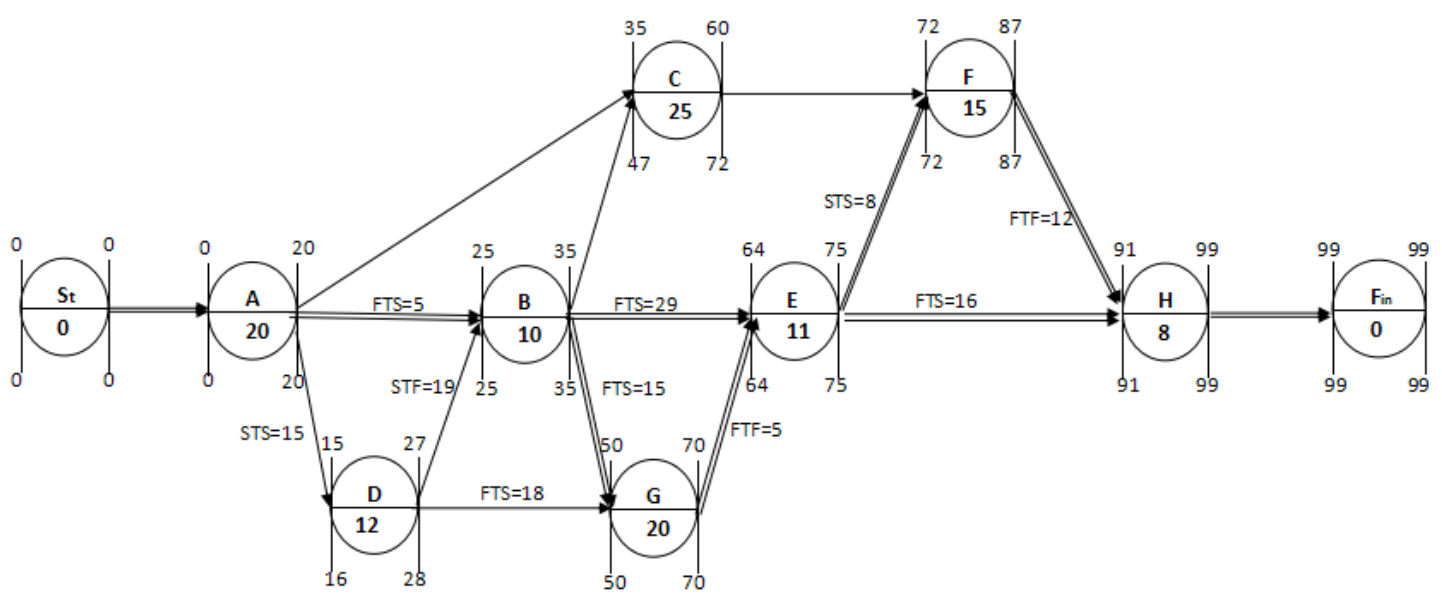

Figure 1. Example of the constant increasing process of the overlapping network plan

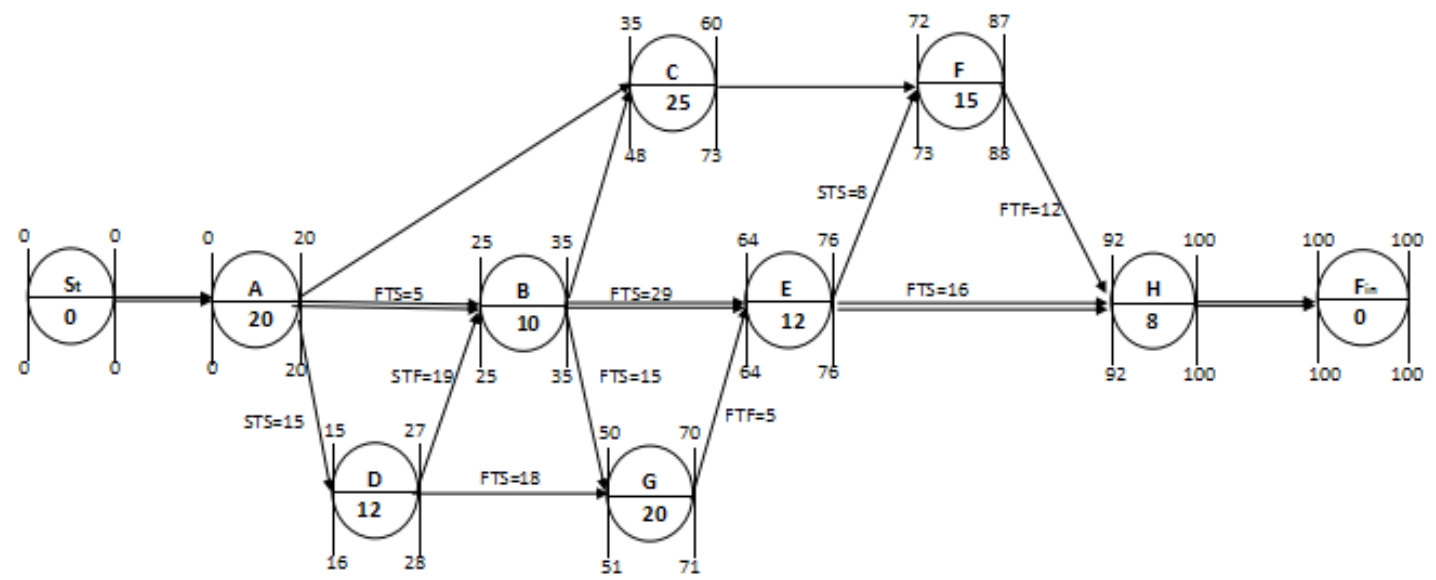

Figure 2. Example of the constant increasing process of the overlapping network plan

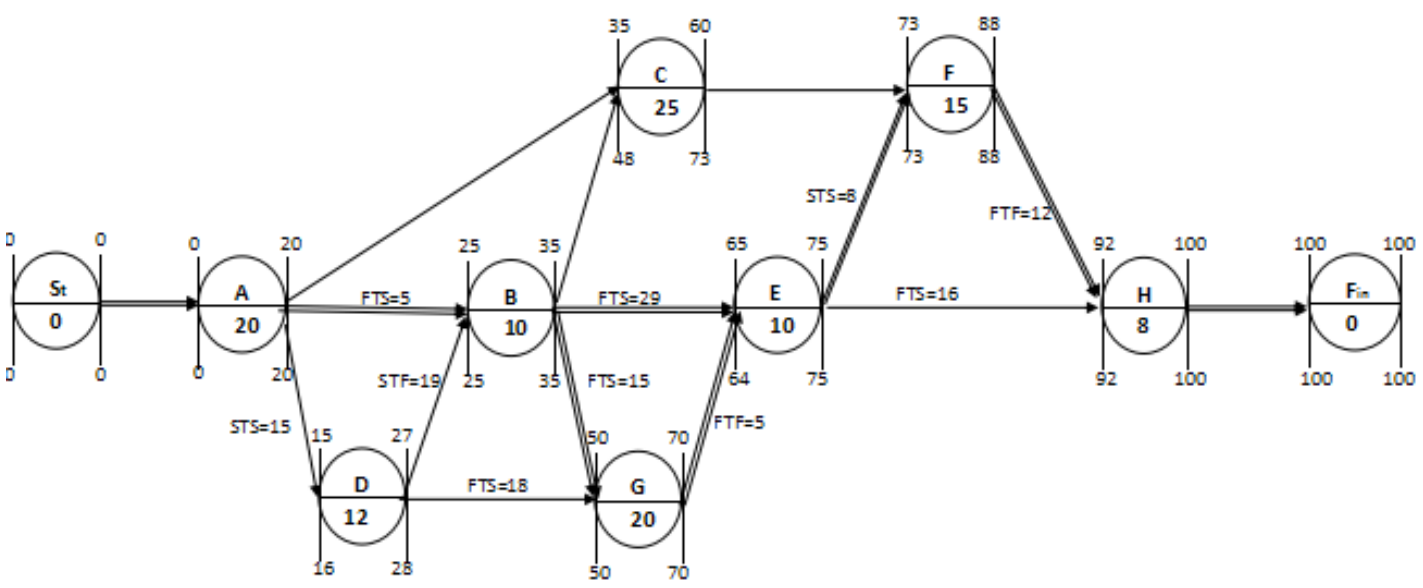

Figure 3. Example of the constant increasing process of the overlapping network plan 


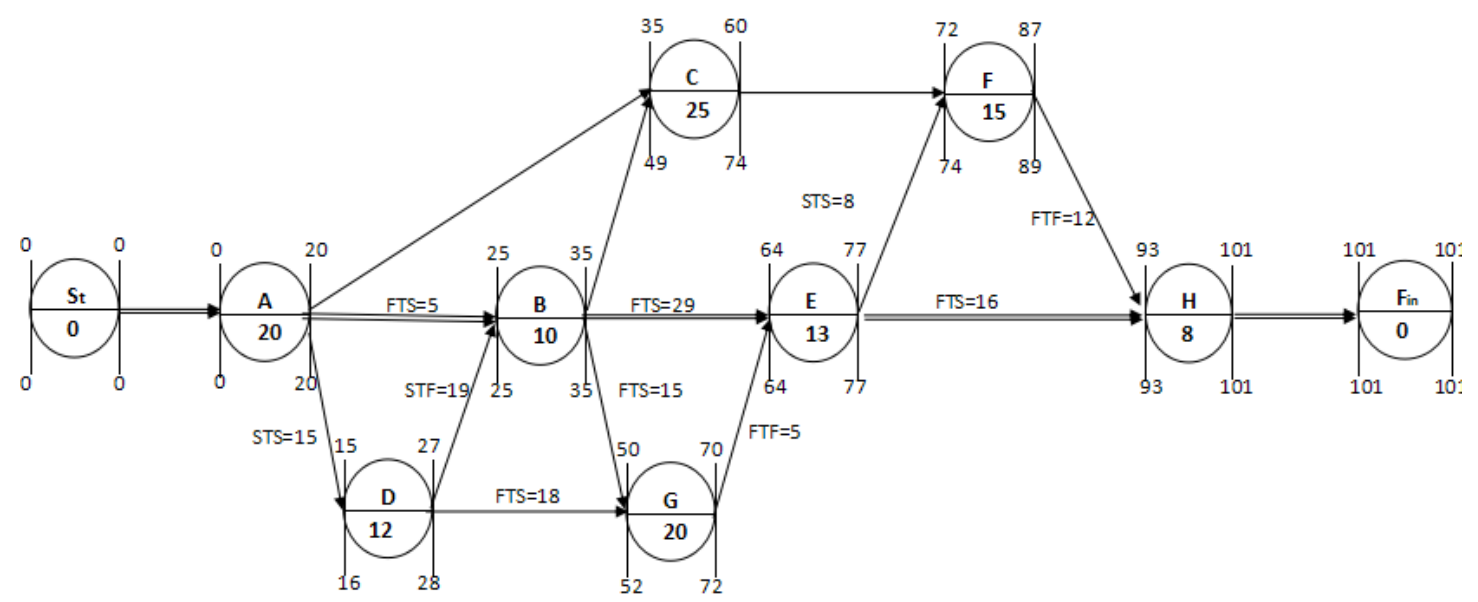

Figure 4. Example of the constant increasing process of the overlapping network plan

From the above cases, we can see the results of the new calculation, the critical path change, the constant increase process is no longer generated

The constant increment procedure exists only in the maximum network constraints, and does not exist in the minimum network constraints. This paper provides a new algorithm for logistics network, which provides a new choice for logistics network planning.

\section{REFERENCES}

[1] Su Zhixiong, Qi Jianxun, Kan Zhinan. New representations and strange phenomenon of spliced networks [J]. Chinese Journal of Systems Engineering-Theory \& Practice, 2015, Vol.35 (1):130-141.

[2] Kan Zhinan, Kong Feng, Qi Jianxun. Research on path lengths paradox and Its characteristics under spliced network [J]. Chinese Journal of Management Science, 2014, Vol.22 (5):121-130.
[3] Kim K, de la Garza J M. Critical path method with multiple calendars [J]. Journal of Construction Engineering Management ASCE, 2005, 131 (3): 330-342.

[4] Chang Chen, Wu Lingfei. Study on the optimization of logistics service pricing for construction projects [J] logistics technology, 2007 eighth: 121-123.

[5] Terry W. Assessing extension of time delays on major prajects [J]. International Journal of Project Management, 2003, 21 (2): 19-26.

[6] Kallantzis A., Lambropoulos S. Correspondence of activity relationships and critical path between time-location diagrams and CPM [J]. Operational Research, 2004, 4 ( 3) : 277-290.

[7] Elmaghraby, S .E. E., J. Kamburowski. The analysis of activity network undergeneralized precedence relations [J]. Management science, 1992, 38 (9): 1245-1263.

[8] Ammar Mohammad A. Float analysis of non-serial repetitive activities [J] Construction Management and Economics, 2003, 21 (5): 535-542. 